\title{
Mesenchymal stromal cells and platelet-rich plasma promote tendon allograft healing in ovine anterior cruciate ligament reconstruction
}

\author{
Adam T. Hexter ${ }^{1,6}$ (D) Anita Sanghani-Kerai ${ }^{1} \cdot$ Nima Heidari $^{2} \cdot$ Deepak M. Kalaskar ${ }^{1} \cdot$ Ashleigh Boyd $^{1}$. \\ Catherine Pendegrass ${ }^{1} \cdot$ Scott A. Rodeo ${ }^{3} \cdot$ Fares S. Haddad ${ }^{4} \cdot$ Gordon W. Blunn $^{5}$
}

Received: 3 September 2020 / Accepted: 24 November 2020 / Published online: 17 December 2020

(c) The Author(s) 2020

\begin{abstract}
Purpose The effect of bone marrow mesenchymal stromal cells (BMSCs) and platelet-rich plasma (PRP) on tendon allograft maturation in a large animal anterior cruciate ligament (ACL) reconstruction model was reported for the first time. It was hypothesised that compared with non-augmented ACL reconstruction, BMSCs and PRP would enhance graft maturation after 12 weeks and this would be detected using magnetic resonance imaging (MRI).

Methods Fifteen sheep underwent unilateral tendon allograft ACL reconstruction using aperture fixation and were randomised into three groups $(n=5)$. Group 1 received 10 million allogeneic BMSCs in $2 \mathrm{ml}$ fibrin sealant; Group 2 received $12 \mathrm{ml} \mathrm{PRP} \mathrm{in} \mathrm{a} \mathrm{plasma} \mathrm{clot} \mathrm{injected} \mathrm{into} \mathrm{the} \mathrm{graft} \mathrm{and} \mathrm{bone} \mathrm{tunnels;} \mathrm{and} \mathrm{Group} 3$ (control) received no adjunctive treatment. At autopsy at 12 weeks, a graft maturation score was determined by the sum for graft integrity, synovial coverage and vascularisation, graft thickness and apparent tension, and synovial sealing at tunnel apertures. MRI analysis ( $n=2$ animals per group) of the signal-noise quotient (SNQ) and fibrous interzone (FIZ) was used to evaluate intra-articular graft maturation and tendon-bone healing, respectively. Spearman's rank correlation coefficient $(r)$ of SNQ, autopsy graft maturation score and bone tunnel diameter were analysed.

Results The BMSC group $(p=0.01)$ and PRP group $(p=0.03)$ had a significantly higher graft maturation score compared with the control group. The BMSC group scored significantly higher for synovial sealing at tunnel apertures $(p=0.03)$ compared with the control group. The graft maturation score at autopsy significantly correlated with the SNQ $(r=-0.83$, $p<0.01)$. The tunnel diameter of the femoral tunnel at the aperture $(r=0.883, p=0.03)$ and mid-portion $(r=0.941, p=0.02)$ positively correlated with the SNQ.

Conclusions BMSCs and PRP significantly enhanced graft maturation, which indicates that orthobiologics can accelerate the biologic events in tendon allograft incorporation. Femoral tunnel expansion significantly correlated with inferior maturation of the intra-articular graft. The clinical relevance of this study is that BMSCs and PRP enhance allograft healing in a translational model, and biological modulation of graft healing can be evaluated non-invasively using MRI.
\end{abstract}

Keywords Anterior cruciate ligament (ACL) reconstruction · Magnetic resonance imaging (MRI) - Autopsy $\cdot$ Bone marrow-derived mesenchymal stromal cells (BMSCs) · Platelet-rich plasma (PRP) · Biological modulation

Adam T. Hexter

a.hexter@ucl.ac.uk

1 Division of Surgery and Interventional Science, University College London (UCL), London, UK

2 Royal London Hospital and Orthopaedic Specialists (OS), London, UK

3 Hospital of Special Surgery (HSS), New York, USA
4 University College London Hospitals, London, UK

5 University of Portsmouth, Portsmouth, UK

6 Institute of Orthopaedics and Musculoskeletal Sciences, Division of Surgery and Interventional Science, Royal National Orthopaedic Hospital, Brockley Hill, Stanmore, London HA7 4LP, UK 


\section{Introduction}

Graft healing after ACL reconstruction consists of tendon-bone healing and matrix remodelling ("ligamentisation") of the intra-articular graft [13, 15]. Graft remodeling can be measured non-invasively using MRI by measuring signal intensity of the intra-articular graft (signal-noise quotient, SNQ) [7]. Similarly, healing at the tendon-bone interface can be evaluated at fibrous interzone (FIZ) on MRI [13]. Bone tunnel widening is a concern [27] and allografts might be associated with increased tunnel widening [3].

There is a growing body of literature exploring biological modulation of graft healing [6]. Mesenchymal stromal cells (MSCs) can enhance tissue regeneration by differentiation, paracrine effects, or via immunomodulatory activity [1]. Platelet-rich plasma (PRP) is a blood derivative that can deliver supraphysiologic doses of cytokines and growth factors [17]. Bone marrow mesenchymal stromal cells (BMSCs) $[12,22]$ and PRP [24] have shown positive effects on graft healing in small animals. Evaluation in a large animal is an important translational step because it permits human-sized grafts and fixation systems to be used [16].

The clinical relevance of this study is that it represents the first large animal ACL reconstruction study to report the effects of BMSCs and PRP on allograft healing. The purpose of this study was to compare the effect of BMSCs and PRP on tendon allograft maturation in a large animal model, and to determine if MRI can be used to identify biological modulation of graft healing. The hypothesis was that BMSCs and PRP would enhance graft maturation after 12 weeks, and graft maturation at autopsy would correlate with graft maturation on MRI.

\section{Materials and methods}

The research was conducted in accordance with a Project License protocol accepted under the UK Home Office Animals (Scientific Procedures) Act 1986 (licence number PF16F4AA0A). This study was approved by the animal warfare review board at the Royal Veterinary College. Fifteen full-mouthed female lowland Mule sheep (age, 2-3 years; weight, $60-75 \mathrm{~kg}$ ) were included. Animals were randomised into three groups ( $n=5$ animals per group). In group 1, 10 million allogeneic BMSCs in fibrin sealant (Baxter, Vienna, Austria) were applied to the graft ( 2 million) and bone tunnels (4 million per tunnel). In group 2, $12 \mathrm{ml}$ of PRP was injected into the graft $(4 \mathrm{ml})$ and the bone tunnels $(4 \mathrm{ml}$ per tunnel). The control animals in Group 3 received no treatment. Post-operatively animals were euthanised after 12 weeks. Graft maturation was examined using MRI and a macroscopic scoring system at autopsy.

\section{PRP preparation}

Autologous PRP was prepared using the Endoret $^{\circledR}\left(\right.$ prgf $\left.^{\circledR}\right)$ Technology (BTI System IV/V; BTI Biotechnology Institute, Vitoria, Spain). $72 \mathrm{ml}$ of venous blood was obtained in $9 \mathrm{ml}$ tubes containing $3.8 \%$ ( $\mathrm{wt} / \mathrm{vol}$ ) sodium citrate. The tubes were centrifuged twice for $8 \mathrm{~min}$ at $580 \mathrm{G}(1902 \mathrm{rpm})$ at room temperature. The $2 \mathrm{ml}$ of plasma located above the buffy coat was collected, with a total PRP volume of $16 \mathrm{ml}$ per animal (Fig. 1a, b). The PRP was activated by adding calcium chloride $(10 \% \mathrm{wt} / \mathrm{vol})$, which led to gel-like transformation within $5 \mathrm{~min}$. The time between venipuncture and surgical delivery was $30 \mathrm{~min}$. In sheep, this technique has

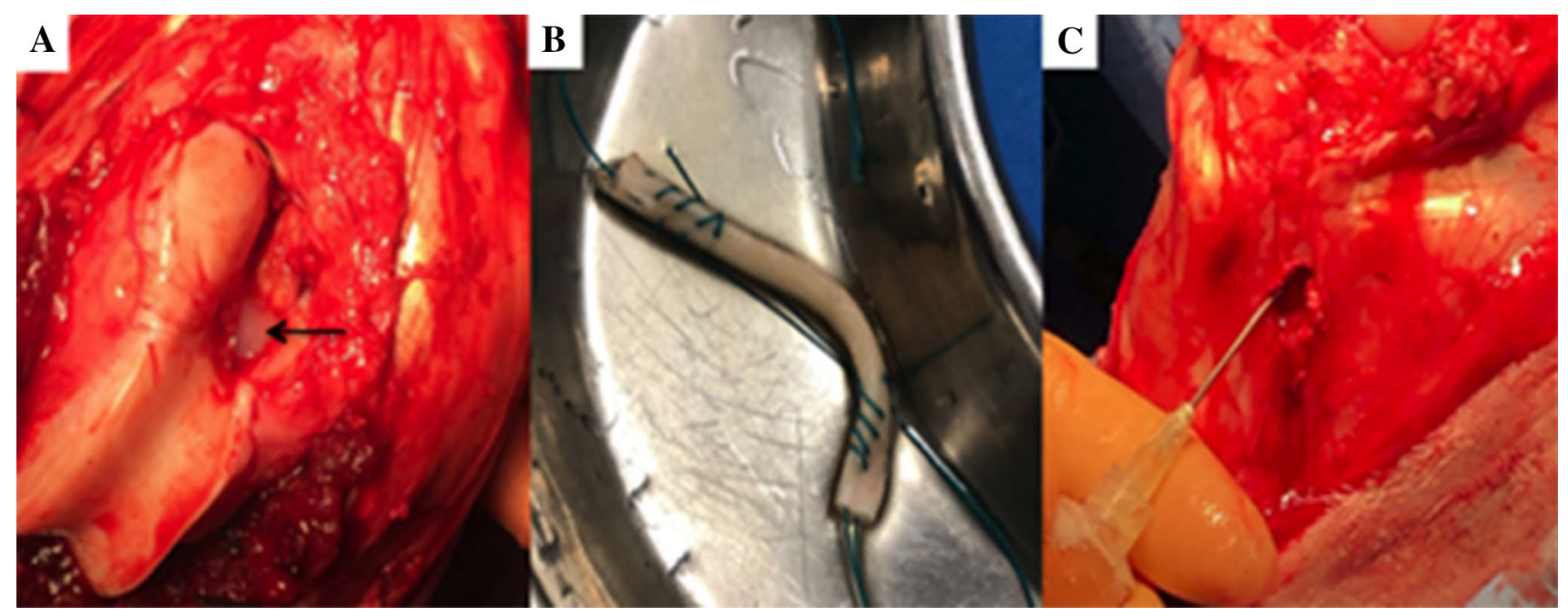

Fig. 1 a Fibrin sealant at femoral aperture (arrow). b SDFT allograft soaking in PRP. c Intra-osseous injection of PRP into tibia 
been shown to yield PRP enriched in platelets and reduced in leucocytes [21].

\section{BMSC harvest}

Bone marrow was aspirated from an adult sheep (age 2 years). $10 \mathrm{ml}$ of bone marrow was aspirated from two regions of the iliac crest into a $20 \mathrm{ml}$ polypropylene tube containing 10,000 International Units (IU) heparin sodium (Wockhardt UK Ltd, Wrexham, UK). The aspirate was combined with standard growth media, which was high glucose Dulbecco's modified Eagle's medium (DMEM) (Invitrogen, Paisley, UK), 10\% FCS (ThermoFisher, Hemel Hempstead, UK) and $1 \%$ penicillin-streptomycin (ThermoFisher). The aspirate was plated onto T-150 culture flasks and cultured at $37{ }^{\circ} \mathrm{C}$ in a $5 \%$ carbon dioxide $\left(\mathrm{CO}_{2}\right)$ incubator. After $72 \mathrm{~h}$, the media was discarded, and fresh growth media was added to supplement the cells that had attached to the flask. The cells were detached with $0.25 \%$ trypsin-EDTA (Gibco, Carlsbad, CA) when they had reached 90\% confluence. The BMSCs were sub-cultured and the growth media was changed every 3 days. 10 million, passage $3-4$ cells were used in each surgical procedure. Tri-lineage potential-adipogenesis, osteogenesis, and chondrogenesis - of the cells was confirmed in vitro prior to surgery.

\section{Superficial digital flexor tendon (SDFT) allograft preparation}

SDFT were harvested from sheep (age 2-3 years) using a previously described technique [8]. The grafts measured $75-85 \mathrm{~mm}$ in length and $7.5-8 \mathrm{~mm}$ in diameter and were sterilised by gamma irradiation at a dose of $25 \mathrm{kGy}$ (Isotron, Reading, UK). The samples were stored at $-20{ }^{\circ} \mathrm{C}$ and thawed at room temperature 30 min before use. The ends of the graft were prepared with a whipstitch using no. 2 Ethibond (Ethicon Inc, Johnson \& Johnson, New Jersey).

\section{Surgical technique}

The right stifle joint was exposed via a medial arthrotomy and the fat pad and ACL were excised. Femoral and tibial bone tunnels (7.8 $\mathrm{mm}$ diameter) were drilled from the ACL footprints through the lateral femoral condyle and anteromedial tibia, respectively. Femoral fixation was achieved using an $8 \times 20 \mathrm{~mm}$ Biosure (Polyether ether ketone, PEEK) interference screw (Smith \& Nephew Endoscopy, Andover, MA). The stifle joint was taken through ten full ranges of motion. Tibial fixation was achieved with the stifle joint in full extension with an $8 \times 25 \mathrm{~mm}$ Biosure PEEK interference screw (Smith \& Nephew Endoscopy, Andover, MA). A tension of $40 \mathrm{~N}$ was applied because this tension has been used in previous ovine studies [10, 11, 30]. In Group 1, $1 \mathrm{~h}$ before surgery, the BMSCs were loaded into $2 \mathrm{ml}$ pre-filled TwoComponent Fibrin Sealant (Baxter, Vienna, Austria). $0.8 \mathrm{ml}$ of fibrin sealant (4 million BMSCs) was added to each bone tunnel and $0.4 \mathrm{ml}$ of fibrin sealant (2 million BMSCs) covered the intra-articular graft (Fig. 1a). In group 2, the graft was infiltrated with $4 \mathrm{ml}$ of activated PRP and left soaking in activated PRP liquid until implantation (Fig. 1b). The interference screws were immersed in $4 \mathrm{ml}$ of activated PRP until implantation. Before graft insertion, the bone in the tunnels was infiltrated with $1 \mathrm{ml}$ PRP at four intervals along the tunnel wall. In total, $4 \mathrm{ml}$ was injected in the femoral tunnel and $4 \mathrm{ml}$ in the tibial tunnel (Fig. 1c). In the control group, the procedure was performed without application of fibrin sealant, BMSCs or PRP. Joint stability was confirmed using an anterior drawer test and the incision was closed in layers. The animals freely mobilised in individual pens for 7 days and thereafter were house as a flock.

\section{Autopsy assessment}

After euthanasia, the joint stability was checked using an anterior drawer test. The hind limb was dissected to examine graft maturation. A scoring system was devised based on parameters used at second-look arthroscopy [9]. A category for synovial sealing at the tunnel apertures was added based on a previously reported scoring system [25]. The scoring system consisted of four main criteria: graft integrity, synovial coverage and vascularisation, graft thickness and apparent tension, and synovial sealing at the tunnel apertures (Table 1). For graft integrity, a complete rupture was defined as complete loss of continuity of all fibres, a "partial

Table 1 Criteria for the Autopsy Graft Maturation Score (out of 9)

\begin{tabular}{|c|c|c|c|c|}
\hline Score & Graft integrity & Synovial coverage and vascularisation & Graft thickness and apparent tension & Synovial sealing at tunnel apertures \\
\hline 3.0 & - & $\geq 75 \%$ with abundant vascularisation & - & - \\
\hline 2.0 & No rupture & $\geq 75 \%$ without vascularisation & $\begin{array}{l}\text { No elongation of a sufficiently thick } \\
\text { graft }\end{array}$ & Circumferential sealing $(>75 \%)$ \\
\hline 1.0 & Partial rupture & $25-74 \%$ & $\begin{array}{l}\text { Partial elongation of a sufficiently } \\
\text { thick graft or no elongation of a } \\
\text { relatively thin graft }\end{array}$ & Moderate synovial sealing $(25-75 \%)$ \\
\hline 0 & Complete rupture & $\leq 25 \%$ & Obvious elongation of a thin graft & Low synovial sealing $(<25 \%)$ \\
\hline
\end{tabular}


rupture" was defined as a loss of continuity of some fibres, and "no rupture" was defined as no loss of fibre continuity. Synovial coverage and vascularisation were assessed by the percentage surface area of the intra-articular graft covered by vascularised synovium. For graft thickness and apparent tension, the highest score was for a thick graft (similar to the ovine ACL) which was not elongated. The second highest mark was for a graft that was either a similar thickness to the ACL and elongated, or not elongated but the graft itself was thin relative to the ACL. The lowest mark was for elongated and thin grafts. For synovial sealing at the tunnel apertures, the highest mark was for circumferential sealing at the aperture ( $>75 \%$ circumference), the second highest mark was for a partial sealing (25-75\% circumference) and the lowest mark was for low sealing $(<25 \%$ circumference). The overall graft maturation score was calculated from the sum of the separate scores (range 4.0-9.0). Two independent researchers blinded to the treatment group scored the grafts.

\section{MRI assessment}

Two animals per group were randomly selected to have MRI scans after post-mortem but before autopsy. The MRI scans were performed using a superconducting 1.5T magnet (Intera Pulsar System, Philips Medical Systems, UK). The stifles were positioned in lateral recumbency with the joint at 90 degrees of flexion. Sequences performed included threedimensional T1 weighted FFE (T1 3D FFE) imaging and fatsaturated proton density (PD-SPIR). Two blinded veterinary radiologists assessed the scans independently using a PACS workstation DICOM viewer (Osirix Imaging Software, version 3.9.2, Bernex, Switzerland).

Radiological assessment of the intra-articular graft was via a scoring system based on the score reported by Howell et al. [7] (Fig. 2) and the signal-to-noise quotient (SNQ). Grade 1 consisted of a graft of homogeneously low signal intensity which was intermediate between the intensity of skeletal muscle and the posterior cruciate ligament (PCL). Grade 2 consisted of a graft of homogeneously low signal intensity in greater than $50 \%$ of the graft, with the remainder of increased signal intensity. Grade 3 was a graft of homogeneously low signal intensity in less than $50 \%$ of the graft with the remainder of increased signal intensity. Grade 4 consisted of a graft of diffusely increased signal intensity. A low graft signal denotes superior graft maturation and, therefore, a lower score denotes better graft healing.

Measurement of the SNQ was performed using a sagittal oblique PD-STIR image. A region of interest (ROI) was placed within the intra-articular graft and within the PCL. For measurement of background signal intensity, a $5 \mathrm{~mm}^{2}$ circular ROI was placed $5 \mathrm{~mm}$ cranial to the skin edge and the mean signal intensity was noted (Fig. 3). The SNQ was measured as follows: $\mathrm{SNQ}=$ (mean graft signal intensity-mean PCL signal intensity)/mean background signal intensity. A lower SNQ denotes more advanced graft maturation.

Graft width was measured on PD-SPIR images on coronal and sagittal images and the average taken. Bone tunnel width was measured using axial slices at the aperture, mid-portion and exit. The precision of measurements was $0.01 \mathrm{~mm}$. Tunnel widening was calculated by subtracting the original drill diameter $(7.8 \mathrm{~mm})$ from the diameter measured after 12 weeks. Radiological assessment of the tendon-bone healing at the fibrous interzone was measured using a peerreviewed three-grade scoring system (Fig. 4) [5]. Grade 1 consisted of a low-intensity signal band in the bone tunnel with no hyper-intense tissue at the tendon-bone interface. Grade 2 consisted of a low-intensity signal band with a partial high-intensity signal band at the interface. In grade 3 , the interface was filled with a continuous high-intensity signal band.

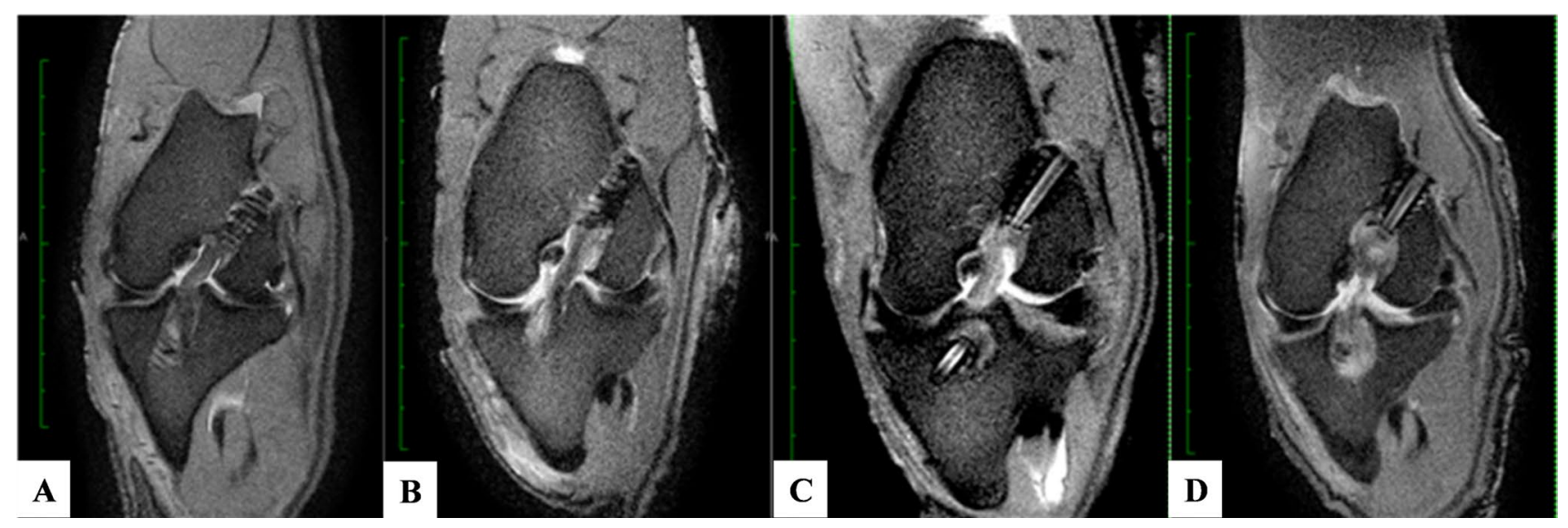

Fig. 2 Scoring grades for intra-articular graft maturity described by Howell et al. [7]. a Grade 1 b Grade 2 c Grade 3 d Grade 4 
Fig. 3 Regions of interest (ROIs) for SNQ Calculation. Blue shows the graft ROI; green circle shows the PCL ROI; and orange shows the background signal
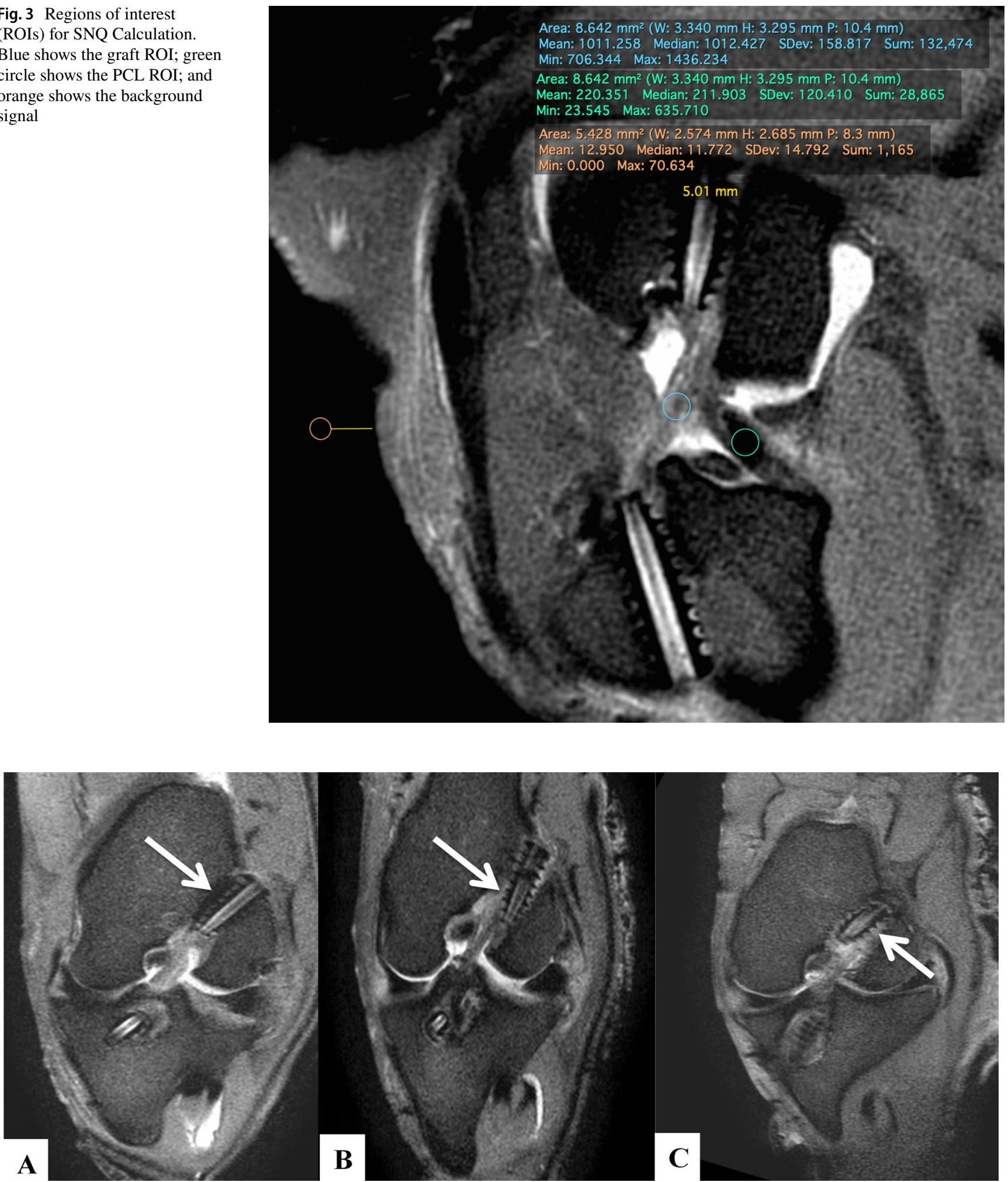

Fig. 4 Scoring grades for tendon-bone healing using femoral tunnel as an example. a Grade 1 b Grade 2 c Grade 3 


\section{Statistical analysis}

IBM SPSS Statistics for Windows, version 26 (IBM Corp., Armonk, N.Y., USA) was used. Data was not normally distributed and reported as medians and interquartile ranges. Mann-Whitney $U$ tests were used to compare treatment groups with the control. Inter-rater reliability as assessed using Kappa statistics. The interpretation was as follows: $<0.40$ was poor, $0.40-0.59$ was fair, $0.60-0.74$ was good, and $\geq 0.74$ was excellent [4]. Correlations between the SNQ, maturation scores and tunnel diameters were examined using Spearman's rank correlation coefficient $(r)$. Significance was assumed at the 0.05 level. Previous animal studies of ACL reconstruction similar to this have used a $n=5$ [6]. Taking a difference in the median average of $15 \%$ and a standard deviation of $10 \%$ with a power of 0.8 and $p$ value of 0.05 , a $n=5$ has been shown to produce significant results.

\section{Results}

\section{Autopsy assessment}

There was no evidence of joint instability or an adverse effect to the treatments. Minimal chondral degeneration and mild synovial inflammation was observed. All the grafts were intact at the time of dissection. The PRP and BMSC treated grafts had remodelled into ACL-like structures. In contrast, grafts from the control group appeared atrophic with minimal vascularisation (Fig. 5).

The kappa score was 0.81 (95\% confidence interval, 0.71-0.91), which is considered excellent. In terms of the overall graft maturation score, the BMSC group $(p=0.01)$ and PRP control $(p=0.03)$ had a significantly higher scores on average compared with the control group (Table 2) (Fig. 6).
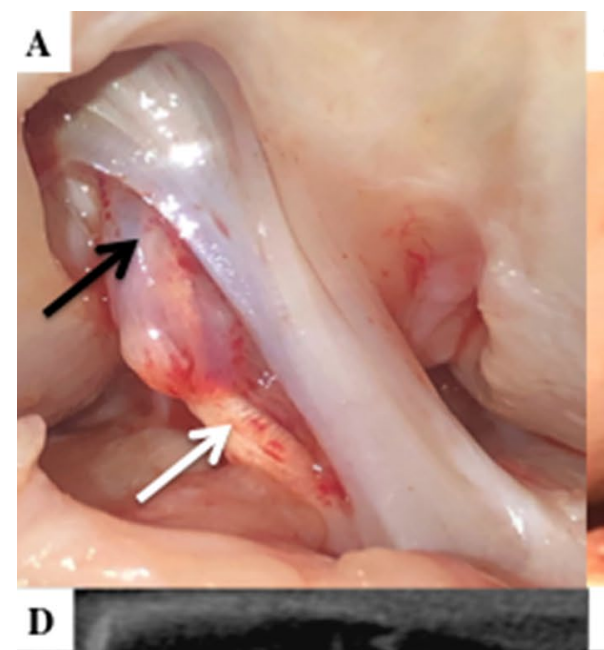

D

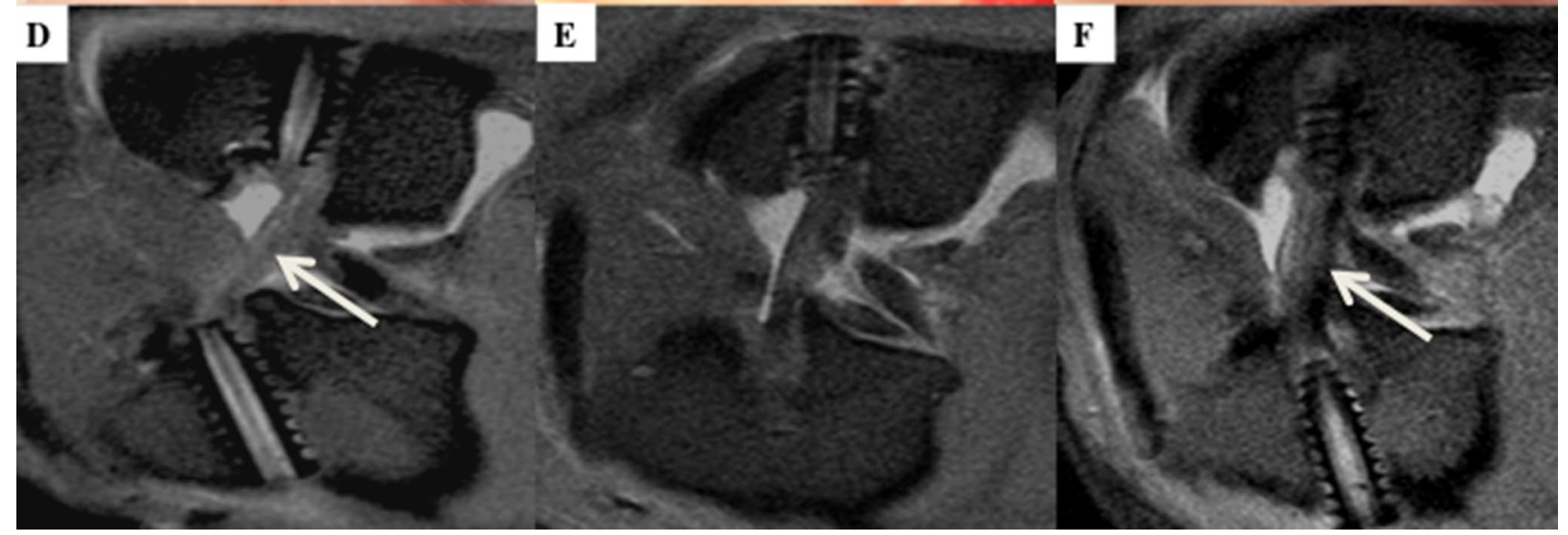

B

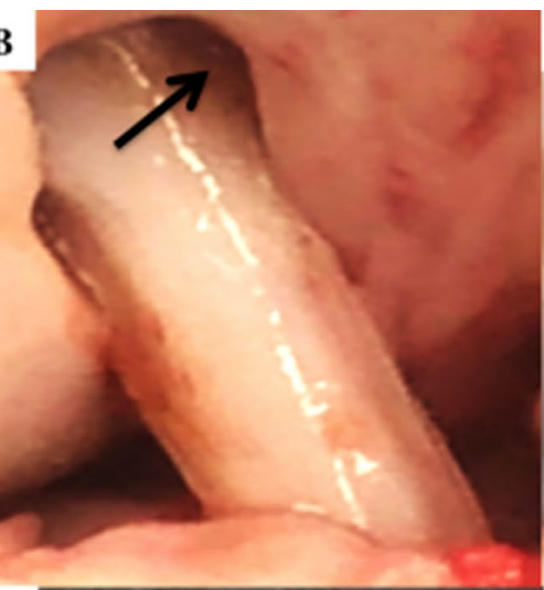

E

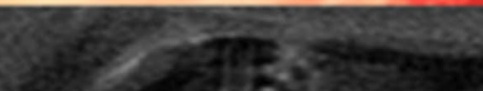

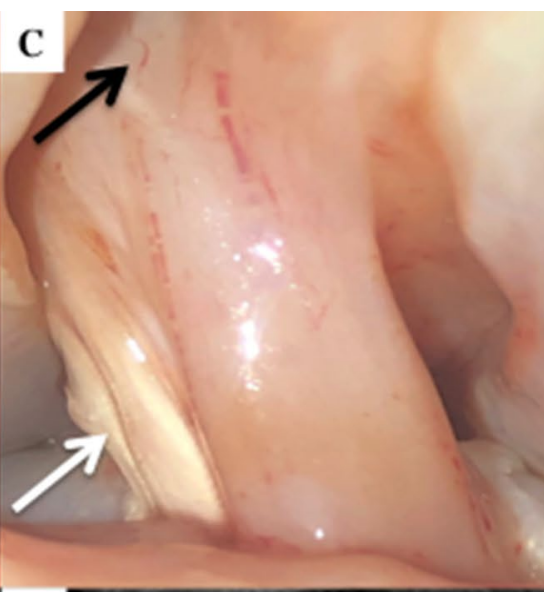

F

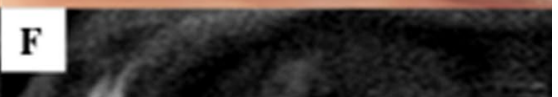

Fig. 5 Autopsy photographs and corresponding sagittal MRI images of control (a, d), PRP (b, e), and BMSC (c, f) group. Autopsy scores, respectively, for graft integrity: synovial coverage: graft thickness/ tension: incorporation at tunnel apertures. $\mathbf{a}=2: 2: 0: 1 ; \mathbf{b}=2: 2: 2: 1$; $\mathbf{c}=2: 3: 2: 2$. In the control group inflammatory tissue is seen between split graft fibres (black arrow). In the PRP group the aperture is not sealed (black arrow) but the aperture is sealed in the BMSC group (black arrow). Regions of the graft appears to be more hypointense in the control and BMSC group (white arrow) but the graft is more homogenous hyperintense in the PRP group 
Table 2 Graft Maturation Score shown as median average (IQR)
Fig. 6 A box and whisker plots showing scores for each variable (showing median and IQR). $n=5$, Mann-Whitney test

\begin{tabular}{llll}
\hline Criteria & Control $(n=5)$ & BMSC $(n=5)$ & PRP $(n=5)$ \\
\hline Graft integrity & $2.0(1.5-2.0)$ & $2.0(2.0-2.0)$ & $2.0(1.5-2.0)$ \\
Synovial coverage and vascularisation & $1.0(1.0-2.0)$ & $2.0(2.0-2.8)$ & $2.5(1.8-2.8)$ \\
Graft thickness and apparent tension & $0.5(0.0-1.0)$ & $1.0(1.0-2.0)$ & $2.0(1.0-2.0)$ \\
Sealing at bone tunnel apertures & $1.0(0.0-1.0)$ & $2.0(1.5-2.0)$ & $1.0(1.0-1.5)$ \\
Overall Graft Maturation Score & $5.0(3.0-5.3)$ & $7.0(7.0-8.3)$ & $6.5(5.8-8.3)$ \\
\hline
\end{tabular}

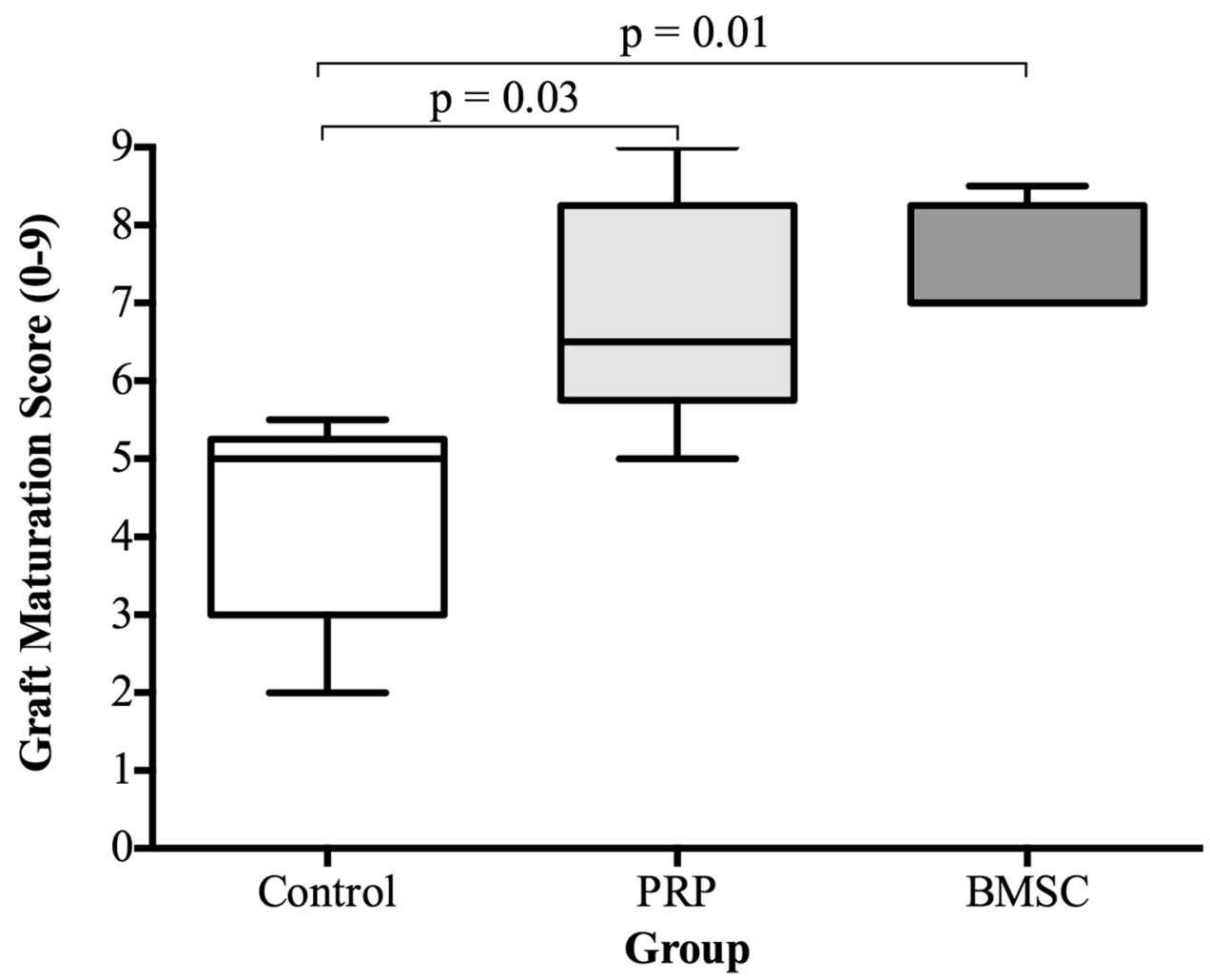

In terms of synovial sealing at tunnel apertures, only the BMSC group had a significantly higher score on average than the control group $(p=0.03)$ (Fig. 7d). No statistically significant differences were seen between the PRP and BMSC groups for the individual variables and total maturation scores.

\section{MRI assessment}

\section{Intra-articular graft}

Graft maturation was higher in BMSC and PRP groups as evidenced by MRI maturation score and SNQ. The average graft diameters in the BMSC and PRP groups were higher than the control group. The intra-articular graft demonstrated higher signal intensity in the control group compared to the PRP and BMSC group.

\section{Tendon-bone healing}

Tendon-bone healing next to the screws was superior to healing at the apertures in all cases in the femur. In the tibia, tendon-bone healing next to the screws was superior to healing at the apertures in all cases except the PRP group where healing was the same at the aperture and adjacent to the screw.

\section{Tunnel widening}

The apertures in the control group were wider than seen in the other two experimental groups, and were filled with hyperintense material (Fig. 3d) (Table 3). 

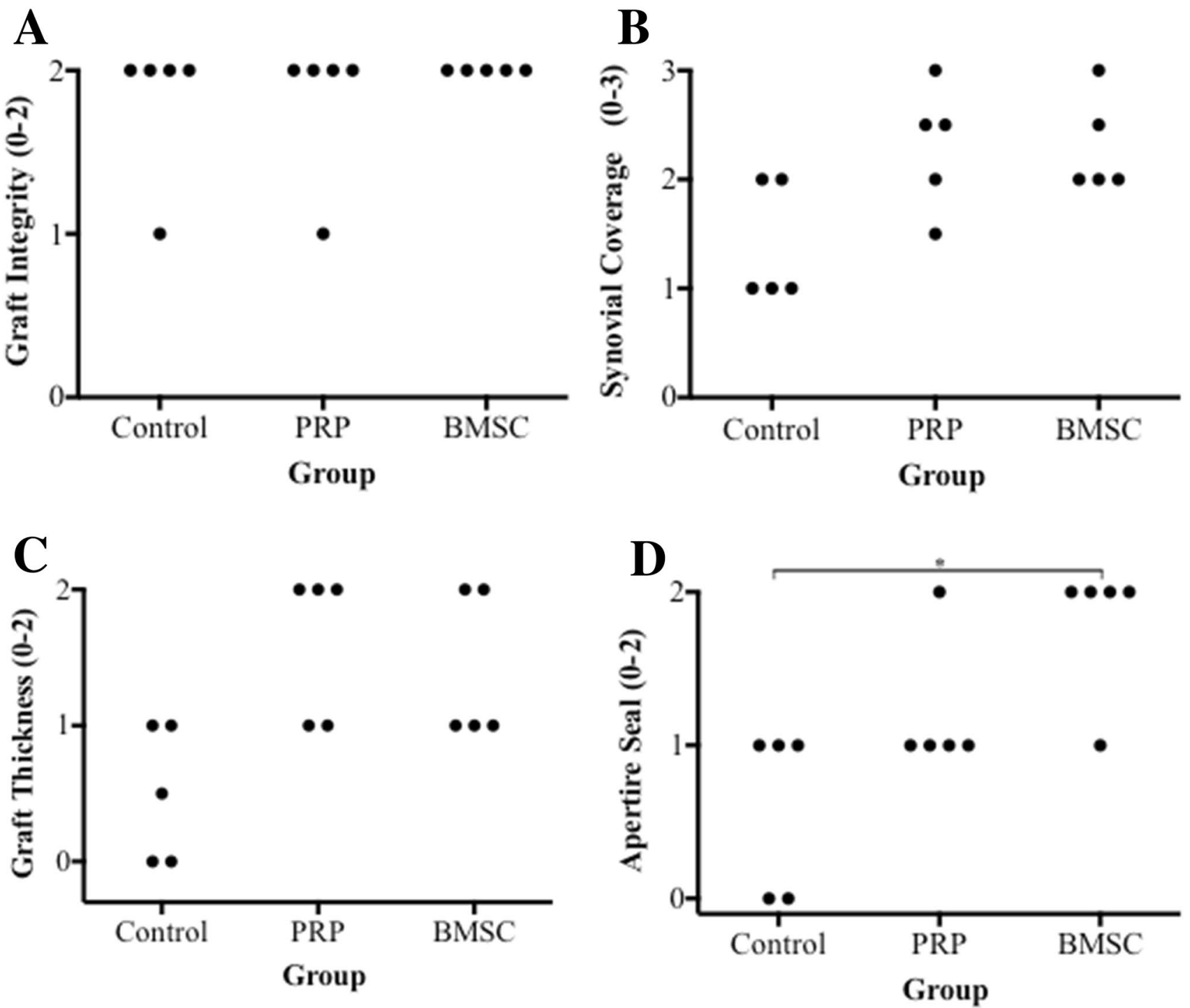

Fig. 7 Dot plots showing scores for each variable. a Graft integrity. b Synovial coverage and vascularisation. c Graft thickness and apparent length. d Incorporation at tunnel apertures. $n=5$, Mann-Whitney test

Table 3 Tunnel widening data $(\mathrm{mm})$

\begin{tabular}{llll}
\hline Tunnel segment & Control $(n=2)$ & BMSC $(n=2)$ & PRP $(n=2)$ \\
\hline Femoral aperture & $3.7(3.2-4.2)$ & $2.7(2.2-3.2)$ & $2.7(2.2-3.2)$ \\
Femoral mid-portion & $1.7(1.2-2.2)$ & $2.7(2.2-3.2)$ & $1.7(1.2-2.2)$ \\
Femoral exit & $3.2(3.2-3.2)$ & $1.7(1.2-2.2)$ & $2.7(2.2-3.2)$ \\
Tibial aperture & $3.7(2.2-5.2)$ & $1.7(1.2-2.2)$ & $1.7(1.2-2.2)$ \\
Tibial mid-portion & $3.2(2.2-4.2)$ & $2.2(2.2-2.2)$ & $2.2(2.2-2.2$ \\
Tibial exit & $1.7(1.2-2.2)$ & $2.2(2.2-2.2$ & $1.2(1.2-1.2)$ \\
\hline
\end{tabular}

Table 4 Correlation analysis of tunnel diameter and signal noise quotient

\begin{tabular}{|c|c|c|c|c|}
\hline \multirow[t]{2}{*}{$\begin{array}{l}\text { Diameter of tunnel } \\
\text { segment }(\mathrm{mm})\end{array}$} & \multicolumn{2}{|c|}{$\begin{array}{l}\text { Signal-noise quotient } \\
\text { (SNQ) }\end{array}$} & \multicolumn{2}{|c|}{$\begin{array}{l}\text { Graft Maturation } \\
\text { Score }(0-9)\end{array}$} \\
\hline & $r$ value & $p$ value & $r$ value & $p$ value \\
\hline Tibia aperture & 0.34 & 0.50 & -0.02 & 0.98 \\
\hline Tibia mid-portion & 0.74 & 0.13 & -0.22 & 0.60 \\
\hline Tibia exit & 0.50 & 0.21 & -0.36 & 0.39 \\
\hline Femoral aperture & 0.88 & $0.03 *$ & -0.70 & 0.08 \\
\hline Femoral midportion & 0.94 & $0.02 *$ & -0.65 & 0.12 \\
\hline Femoral exit & 0.44 & 0.40 & -0.34 & 0.20 \\
\hline
\end{tabular}

$* p<0.05$ 


\section{Correlation between autopsy and MRI graft maturations score}

The autopsy graft maturation scores and the MRI graft maturation score were significantly inversely correlated $(r=-0.83, p \leq 0.01)$.

\section{Correlation between SNQ and Tunnel Segment Diameter.}

The SNQ was positively correlated with the femoral bone tunnel diameter at the aperture $(r=0.88, p=0.03)$ and midportion $(r=0.94, p=0.02)$. No significant correlations were seen between the tibial tunnel diameter and SNQ (Table 4).

\section{Discussion}

Our first hypothesis that BMSCs and PRP would enhance graft maturation after 12 weeks was verified through significant improvements in autopsy graft maturation scores. Our second hypothesis that graft maturation at autopsy would correlate with graft maturation measured on magnetic resonance imaging (MRI) was also verified.

BMSCs have potential to modulate graft healing because of their capacity for multi-lineage differentiation and diverse paracrine effects [6]. This study supports evidence from small animals that BMSCs can enhance ACL graft healing $[14,22]$. The manner by which BMSCs exert their effect on graft healing remains unknown, and future studies that tracks the cells after implantation will help reveal the mechanism. The decision to use allogeneic cells in this study rather than autologous cells was because allogeneic therapy is a more scalable treatment option due to lower processing costs [18]. A disadvantage of allogeneic cells is a higher risk of immune rejection but in this study no signs of adverse inflammatory effects were seen in this study, which is an important finding. Tunnel widening at the aperture [27] is common and is associated with synovial fluid influx [23]. An important finding of our study was that BMSCs were associated with higher level of sealing at the tunnel apertures, which might in turn reduce synovial fluid influx.

The effect of local PRP application from coated sutures has been reported in sheep [28] but to our knowledge no study has examined the effect of intra-tendinous and intraosseous injection of PRP on tendon allograft healing. In this study, PRP treatment led to significantly enhanced graft maturation of allografts at autopsy and MRI. Nevertheless, currently there is no level 1 evidence that shows a benefit of PRP in ACL reconstruction [2]. One reason for this could be the wide variation in the PRP administration techniques because some studies inject PRP just into the graft [19], whereas others inject into the bone tunnels. To maximise exposure of the tendon-bone interface to the PRP, we recommend both intra-osseous and intra-tendinous injection [20].

MRI is a popular technique to evaluate ACL graft maturity after surgical reconstruction [26]. We demonstrated that MRI is a useful tool to measure biological modulation of graft healing because autopsy graft maturation scores correlated with MRI graft maturity scores. Zhang et al. [31] showed for the first time that graft signal intensity correlates with radius of the femoral tunnel aperture. This study corroborate these findings because we observed that SNQ correlated with the diameter of the femoral tunnel aperture and mid-portion. There is growing evidence that maturation of the intra-articular graft is related to tunnel widening in the femoral tunnel.

This study has several limitations. First, graft healing is a complex process that occurs over many months [29] but our analysis was at a single time point. Second, the number of animals included in the study was small, especially for the MRI analysis. Nevertheless, the purpose of this proof-ofconcept study was to determine if MRI can detect biological modulation of graft healing and future MRI studies are now required with larger sample sizes that permit statistical comparisons. Third, histological analysis was not reported and therefore correlation of histological findings with MRI is an important area of future research. Fourth, the joint stability was not quantified using a rolimeter and biomechanical testing was not performed. Finally, fibrin sealant alone might have a positive effect by sealing the aperture at the time of surgery [23] and a better study design would have included an additional group that received fibrin sealant alone.

\section{Conclusion}

BMSCs and PRP significantly enhanced tendon allograft maturation after 12 weeks in a translational large animal model compared to an untreated control. MRI could be used to non-invasively examine biological augmentation of ACL graft healing in clinical practice.

Author contributions AH, University College London: lead researcher and surgeon, wrote the original manuscript before edits, performed surgeries and autopsy analysis with Gordon W. Blunn. AS-K, University College London: assisted in the in vitro stem cells research and stem cell surgical application, edited the manuscript prior to submission. NH, Royal London Hospital: assisted in the PRP application and edited the manuscript prior to submission. DK, University College London: reviewed and edited the manuscript prior to submission (especially the PRP methodology). AB, University College London: contributed to writing the manuscript and reviewed the manuscript prior to submission (especially the stem cell methodology). CP, University College London: edited the manuscript prior to submission. SR, Hospital of 
Special Surgery, NYC: contributed in a large way to the final manuscript, especially discussion and interpretation of findings and animal model design. FH, University College London Hospitals: contributed to writing the manuscript and reviewed the manuscript prior to submission. GB, University of Portsmouth: senior author, supervision of the planning and writing of the manuscript, and reviewed the manuscript prior to submission.

Funding Royal College of Surgeons Research Fellowship $£ 54,885$, Biotechnology Institute $£ 23,200$, Royal Free Charity $£ 9500$ Project Grant.

\section{Compliance with ethical standards}

Conflict of interest The author declare that they have no conflict of interest.

\section{Ethical approval Yes.}

Open Access This article is licensed under a Creative Commons Attribution 4.0 International License, which permits use, sharing, adaptation, distribution and reproduction in any medium or format, as long as you give appropriate credit to the original author(s) and the source, provide a link to the Creative Commons licence, and indicate if changes were made. The images or other third party material in this article are included in the article's Creative Commons licence, unless indicated otherwise in a credit line to the material. If material is not included in the article's Creative Commons licence and your intended use is not permitted by statutory regulation or exceeds the permitted use, you will need to obtain permission directly from the copyright holder. To view a copy of this licence, visit http://creativecommons.org/licenses/by/4.0/.

\section{References}

1. Caplan AI (2017) Mesenchymal stem cells: time to change the name! Stem Cells Transl Med 6:1445-1451

2. Davey MS, Hurley ET, Withers D, Moran R, Moran CJ (2020) Anterior cruciate ligament reconstruction with platelet-rich plasma: a systematic review of randomized control trials. Arthroscopy. https://doi.org/10.1016/j.arthro.2019.11.004

3. Fahey M, Indelicato PA (1994) Bone tunnel enlargement after anterior cruciate ligament replacement. Am J Sports Med $22: 410-414$

4. Fleiss JL (1999) Reliability of measurement. In: Fleiss JL (ed) The design and analysis of clinical experiments. https://doi. org/10.1002/9781118032923.ch1. Accessed 12 Dec 2020

5. Ge Y, Li H, Tao H, Hua Y, Chen J, Chen S (2015) Comparison of tendon-bone healing between autografts and allografts after anterior cruciate ligament reconstruction using magnetic resonance imaging. Knee Surg Sports Traumatol Arthrosc 23:954-960

6. Hexter AT, Thangarajah T, Blunn G, Haddad FS (2018) Biological augmentation of graft healing in anterior cruciate ligament reconstruction: a systematic review. Bone Joint J 100:271-284

7. Howell SM, Clark JA, Blasier RD (1991) Serial magnetic resonance imaging of hamstring anterior cruciate ligament autografts during the first year of implantation: a preliminary study. Am J Sports Med 19:42-47

8. Hunt P, Scheffler SU, Unterhauser FN, Weiler A (2005) A model of soft-tissue graft anterior cruciate ligament reconstruction in sheep. Arch Orthop Trauma Surg 125:238-248
9. Kim S-G, Jung JH, Song J-H, Bae J-H (2019) Evaluation parameters of graft maturation on second-look arthroscopy following anterior cruciate ligament reconstruction: a systematic review. Knee Surg Relat Res 31:2

10. Kondo E, Yasuda K, Katsura T, Hayashi R, Azuma C, Tohyama H (2011) Local administration of autologous synovium-derived cells improve the structural properties of anterior cruciate ligament autograft reconstruction in sheep. Am J Sports Med 39:999-1007

11. Kondo E, Yasuda K, Katsura T, Hayashi R, Kotani Y, Tohyama H (2012) Biomechanical and histological evaluations of the doubled semitendinosus tendon autograft after anterior cruciate ligament reconstruction in sheep. Am J Sports Med 40:315-324

12. Li F, Jia H, Yu C (2007) ACL reconstruction in a rabbit model using irradiated Achilles allograft seeded with mesenchymal stem cells or PDGF-B gene-transfected mesenchymal stem cells. Knee Surg Sports Traumatol Arthrosc 15:1219-1227

13. Li H-Y, Li H, Wu Z-Y, Chen J-W, Chen S-Y (2018) MRI-based tendon bone healing is related to the clinical functional scores at the first year after anterior cruciate ligament reconstruction with hamstring tendon autograft. Knee Surg Sports Traumatol Arthrosc 26:615-621

14. Lim JK, Hui J, Li L, Thambyah A, Goh J, Lee EH (2004) Enhancement of tendon graft osteointegration using mesenchymal stem cells in a rabbit model of anterior cruciate ligament reconstruction. Arthroscopy 20:899-910

15. Liu S, Li H, Tao H, Sun Y, Chen S, Chen J (2018) A randomized clinical trial to evaluate attached hamstring anterior cruciate ligament graft maturity with magnetic resonance imaging. Am J Sports Med 46:1143-1149

16. Madry H, Ochi M, Cucchiarini M, Pape D, Seil R (2015) Large animal models in experimental knee sports surgery: focus on clinical translation. J Exp Orthop 2:9

17. Marx RE, Carlson ER, Eichstaedt RM, Schimmele SR, Strauss JE, Georgeff KR (1998) Platelet-rich plasma: growth factor enhancement for bone grafts. Oral Surg Oral Med Oral Pathol Oral Radiol Endod 85:638-646

18. Pigeau GM, Csaszar E, Dulgar-Tulloch A (2018) Commercial scale manufacturing of allogeneic cell therapy. Front Med 5:233

19. Radice F, Yánez R, Gutiérrez V, Rosales J, Pinedo M, Coda S (2010) Comparison of magnetic resonance imaging findings in anterior cruciate ligament grafts with and without autologous platelet-derived growth factors. Arthroscopy 26:50-57

20. Sánchez M, Anitua E, Azofra J, Prado R, Muruzabal F, Andia I (2010) Ligamentization of tendon grafts treated with an endogenous preparation rich in growth factors: gross morphology and histology. Arthroscopy 26:470-480

21. Sánchez M, Anitua E, Delgado D, Prado R, Sánchez P, Fiz N et al (2017) Ultrasound-guided plasma rich in growth factors injections and scaffolds hasten motor nerve functional recovery in an ovine model of nerve crush injury. J Tissue Eng Regen Med 11:1619-1629

22. Soon MYH, Hassan A, Hui JHP, Goh JCH, Lee EH (2007) An analysis of soft tissue allograft anterior cruciate ligament reconstruction in a rabbit model: a short-term study of the use of mesenchymal stem cells to enhance tendon osteointegration. Am J Sports Med 35:962-971

23. Sun L, Zhou X, Wu B, Tian M (2012) Inhibitory effect of synovial fluid on tendon-to-bone healing: an experimental study in rabbits. Arthroscopy 28:1297-1305

24. Teng C, Zhou C, Xu D, Bi F (2016) Combination of platelet-rich plasma and bone marrow mesenchymal stem cells enhances tendon-bone healing in a rabbit model of anterior cruciate ligament reconstruction. J Orthop Surg Res 11:96

25. Uchio Y, Ochi M, Adachi N, Kawasaki K, Kuriwaka M (2003) Determination of time of biologic fixation after anterior cruciate 
ligament reconstruction with hamstring tendons. Am J Sports Med 31:345-352

26. Van Dyck P, Zazulia K, Smekens C, Heusdens CHW, Janssens T, Sijbers J (2019) Assessment of anterior cruciate ligament graft maturity with conventional magnetic resonance imaging: a systematic literature review. Orthop J Sports Med 7:2325967119849012

27. Weber AE, Delos D, Oltean HN, Vadasdi K, Cavanaugh J, Potter HG et al (2015) Tibial and femoral tunnel changes after ACL reconstruction: a prospective 2-year longitudinal MRI study. Am J Sports Med 43:1147-1156

28. Weiler A, Forster C, Hunt P, Falk R, Jung T, Unterhauser FN et al (2004) The influence of locally applied platelet-derived growth factor-BB on free tendon graft remodeling after anterior cruciate ligament reconstruction. Am J Sports Med 32:881-891

29. Weiler A, Peters G, Maurer J, Unterhauser FN, Sudkamp NP (2001) Biomechanical properties and vascularity of an anterior cruciate ligament graft can be predicted by contrast-enhanced magnetic resonance imaging. A two-year study in sheep. Am J Sports Med 29:751-761
30. Yoshikawa T, Tohyama H, Katsura T, Kondo E, Kotani Y, Matsumoto $\mathrm{H}$ et al (2006) Effects of local administration of vascular endothelial growth factor on mechanical characteristics of the semitendinosus tendon graft after anterior cruciate ligament reconstruction in sheep. Am J Sports Med 34:1918-1925

31. Zhang S, Liu S, Yang L, Chen S, Chen S, Chen J (2020) Morphological changes of the femoral tunnel and their correlation with hamstring tendon autograft maturation up to 2 years after anterior cruciate ligament reconstruction using femoral cortical suspension. Am J Sports Med 48:554-564

Publisher's Note Springer Nature remains neutral with regard to jurisdictional claims in published maps and institutional affiliations. 